\title{
Kaczmarz algorithm in Hilbert space
}

\author{
by \\ Rainis Haller (Tartu) and Ryszard Szwarc (Wrocław)
}

\begin{abstract}
The aim of the Kaczmarz algorithm is to reconstruct an element in a Hilbert space from data given by inner products of this element with a given sequence of vectors. The main result characterizes sequences of vectors leading to reconstruction of any element in the space. This generalizes some results of Kwapień and Mycielski.
\end{abstract}

1. Introduction. Let $\left\{e_{n}\right\}_{n=0}^{\infty}$ be a sequence of unit vectors in a Hilbert space $\mathcal{H}$. For a given $x \in \mathcal{H}$ we have the numbers $\left\{\left\langle x, e_{n}\right\rangle\right\}_{n=0}^{\infty}$. We want to reconstruct $x$ from these numbers. The sequence $\left\{e_{n}\right\}_{n=0}^{\infty}$ should be linearly dense. Define

$$
\begin{aligned}
x_{0} & =\left\langle x, e_{0}\right\rangle e_{0}, \\
x_{n} & =x_{n-1}+\left\langle x-x_{n-1}, e_{n}\right\rangle e_{n} .
\end{aligned}
$$

We are interested in when $x_{n} \rightarrow x$ for any $x \in \mathcal{H}$. The sequences $\left\{e_{n}\right\}_{n=0}^{\infty}$ for which this holds will be called effective.

The formula is called the Kaczmarz algorithm. In 1937 Kaczmarz (see [1]) considered this problem in the finite-dimensional case. He proved that if $\operatorname{dim} \mathcal{H}<\infty$ and the sequence $\left\{e_{n}\right\}_{n=0}^{\infty}$ is linearly dense and periodic then it is effective.

Let $P_{n}$ be the orthogonal projection onto $e_{n}^{\perp}$. Then we have

$$
\begin{aligned}
x_{n} & =x_{n-1}+\left(I-P_{n}\right)\left(x-x_{n-1}\right), \\
x-x_{n} & =P_{n}\left(x-x_{n-1}\right), \\
x-x_{n} & =P_{n} P_{n-1} \cdots P_{1} P_{0} x .
\end{aligned}
$$

Therefore the sequence $\left\{e_{n}\right\}_{n=0}^{\infty}$ is effective if and only if the operators $P_{n} P_{n-1} \cdots P_{1} P_{0}$ tend to zero strongly. Since the norms of these operators are bounded it suffices to get pointwise convergence on a linearly dense subset of vectors, e.g. on members of the sequence $\left\{e_{n}\right\}_{n=0}^{\infty}$.

2000 Mathematics Subject Classification: Primary 41A65.

Key words and phrases: Kaczmarz algorithm, Hilbert space.

Supported by Research Training Network "Harmonic Analysis and Related Problems" Contract HPRN-CT-2001-00273. The authors were also supported by the Estonian Science Foundation, Grant no. 4400 and KBN (Poland), Grant 2 P03A 028 25, respectively. 
The Kaczmarz theorem can now be proved as follows. Let $\operatorname{dim} \mathcal{H}<\infty$ and $\left\{e_{n}\right\}_{n=0}^{\infty}$ be $N$-periodic. For $A=P_{N-1} \cdots P_{1} P_{0}$ it suffices to show that $A^{n}$ tends to zero. We claim that $\|A\|<1$. If not, there is a vector $x$ such that $\|A x\|=\|x\|=1$. Then $\left\|P_{0} x\right\| \geq\|A x\|=\|x\|$, hence $P_{0} x=x$. Similarly $P_{1} x=x, \ldots, P_{N-1} x=x$, which implies that $x \perp e_{0}, e_{1}, \ldots, e_{N-1}$. Since the vectors $\left\{e_{n}\right\}_{n=0}^{N-1}$ are linearly dense we get $x=0$.

We now turn to the infinite-dimensional case. We recall some basic properties of the algorithm which can be found in [2]. By construction the vector $x_{n}$ is a linear combination of $e_{0}, e_{1}, \ldots, e_{n}$. It can be shown that

$$
x_{n}=\sum_{i=0}^{n}\left\langle x, g_{i}\right\rangle e_{i},
$$

where the vectors $g_{n}$ are given by the recurrence relation

$$
g_{0}=e_{0}, \quad g_{n}=e_{n}-\sum_{i=0}^{n-1}\left\langle e_{n}, e_{i}\right\rangle g_{i}
$$

or

$$
\sum_{i=0}^{n} m_{n i} g_{i}=e_{n}, \quad m_{n i}=\left\langle e_{n}, e_{i}\right\rangle .
$$

By (2) we have

$$
x-x_{n-1}=x-x_{n}+\left\langle x, g_{n}\right\rangle e_{n} .
$$

Since by (1) the vectors $x-x_{n}$ and $e_{n}$ are orthogonal we get

$$
\begin{aligned}
\|x\|^{2} & =\left\|x-x_{0}\right\|^{2}+\left|\left\langle x, g_{0}\right\rangle\right|^{2}, \\
\left\|x-x_{n-1}\right\|^{2} & =\left\|x-x_{n}\right\|^{2}+\left|\left\langle x, g_{n}\right\rangle\right|^{2}, \quad n \geq 1 .
\end{aligned}
$$

Summing up these equalities gives

$$
\|x\|^{2}-\lim _{n \rightarrow \infty}\left\|x-x_{n}\right\|^{2}=\sum_{n=0}^{\infty}\left|\left\langle x, g_{n}\right\rangle\right|^{2} .
$$

Therefore the sequence $\left\{e_{n}\right\}_{n=0}^{\infty}$ is effective if and only if

$$
\|x\|^{2}=\sum_{n=0}^{\infty}\left|\left\langle x, g_{n}\right\rangle\right|^{2} \quad \text { for any } x \in \mathcal{H} .
$$

This equation means that $\left\{g_{n}\right\}_{n=0}^{\infty}$ is a tight frame with constant 1 as was already mentioned in [2]. We have noticed before that it suffices to check formula (5) on vectors $\left\{e_{n}\right\}_{n=0}^{\infty}$, provided they form a linearly dense subset in $\mathcal{H}$. 
2. Characterization of effective sequences. The formula (3) implies that $g_{n}$ is a linear combination of the vectors $e_{0}, e_{1}, \ldots, e_{n}$, i.e.

$$
\sum_{i=0}^{n-1} c_{n i} e_{i}+e_{n}=g_{n}
$$

for some coefficients $c_{n j}$. By (4) these coefficients can be obtained by taking the algebraic inverse matrix to the lower triangular matrix $I+M$ where

$$
M=\left(\begin{array}{cccccc}
0 & 0 & 0 & 0 & 0 & \ldots \\
m_{10} & 0 & 0 & 0 & 0 & \ldots \\
m_{20} & m_{21} & 0 & 0 & 0 & \ldots \\
m_{30} & m_{31} & m_{32} & 0 & 0 & \ldots \\
\vdots & \vdots & \vdots & \vdots & \ddots & \ddots
\end{array}\right), \quad m_{i j}=\left\langle e_{i}, e_{j}\right\rangle .
$$

Namely $(I+M)^{-1}=I+U$, where

$$
U=\left(\begin{array}{cccccc}
0 & 0 & 0 & 0 & 0 & \ldots \\
c_{10} & 0 & 0 & 0 & 0 & \ldots \\
c_{20} & c_{21} & 0 & 0 & 0 & \ldots \\
c_{30} & c_{31} & c_{32} & 0 & 0 & \ldots \\
\vdots & \vdots & \vdots & \vdots & \ddots & \ddots
\end{array}\right)
$$

The matrix $U$ and the coefficients $c_{n i}$ will play a crucial role in all what follows. Since the matrices $I+M$ and $I+U$ are inverse to each other we get

$$
\begin{aligned}
U M & =M U=-M-U, \\
U^{*} M^{*} & =M^{*} U^{*}=-M^{*}-U^{*} .
\end{aligned}
$$

Our first simple result shows that the matrix $U$ is a contraction in the Hilbert space $\ell^{2}\left(\mathbb{N}_{0}\right)$.

Proposition 1. Let $U$ and $M$ be strictly lower triangular matrices such that $M U=U M=-U-M$. Then $U$ is a contraction if and only if the matrix $M+M^{*}+I$ is positive definite. In that case there is a Hilbert space $\mathcal{H}$ and vectors $\left\{e_{n}\right\}_{n=0}^{\infty}$ in $\mathcal{H}$ such that $M+M^{*}+I$ is the Gram matrix of these vectors.

Proof. Let $M_{n}$ and $U_{n}$ denote the truncated matrices given by

$$
M_{n}=\left(\begin{array}{cccc}
0 & & & \\
m_{10} & 0 & & \\
\vdots & \ddots & 0 & \\
m_{n 0} & \cdots & m_{n, n-1} & 0 \\
0 & \cdots & 0 & \ddots \\
\vdots & \vdots & \vdots & \vdots
\end{array}\right), \quad U_{n}=\left(\begin{array}{ccccc}
0 & & & \\
c_{10} & 0 & & \\
\vdots & \ddots & 0 & \\
c_{n 0} & \cdots & c_{n, n-1} & 0 \\
0 & \cdots & 0 & \ddots \\
\vdots & \vdots & \vdots & \vdots
\end{array}\right) .
$$


Then $M_{n}$ and $U_{n}$ are bounded on $\ell^{2}\left(\mathbb{N}_{0}\right)$ and by assumption $M_{n} U_{n}=$ $U_{n} M_{n}=-U_{n}-M_{n}$. Assume the matrix $M+M^{*}+I$ is positive definite. Then the matrix $M_{n}+M_{n}^{*}+I$ corresponds to a positive bounded operator on $\ell^{2}\left(\mathbb{N}_{0}\right)$. Thus

$$
0 \leq\left(U_{n}^{*}+I\right)\left(M_{n}+M_{n}^{*}+I\right)\left(U_{n}+I\right)=I-U_{n}^{*} U_{n} .
$$

Hence $\left\|U_{n}\right\| \leq 1$, where $\|\cdot\|$ denotes the operator norm. Consequently, we obtain $\|U\| \leq 1$. The converse implication follows from

$$
\left(M_{n}^{*}+I\right)\left(I-U_{n}^{*} U_{n}\right)\left(M_{n}+I\right)=M_{n}+M_{n}^{*}+I .
$$

Indeed, if $\|U\| \leq 1$ then $\left\|U_{n}\right\| \leq 1$. Therefore the matrix $M_{n}+M_{n}^{*}+I$ is positive definite, which implies that so also is $M+M^{*}+I$. It is then well known that there exist a Hilbert space $\mathcal{H}$ and vectors $\left\{e_{n}\right\}_{n=0}^{\infty}$ such that

$$
m_{i j}=\left\langle e_{i}, e_{j}\right\rangle, \quad i>j .
$$

Now we can state the main result of our paper.

THEOREM 1. The sequence $\left\{e_{n}\right\}_{n=0}^{\infty}$ is effective if and only if it is linearly dense and $U$ is a partial isometry, i.e. $U^{*} U$ is an orthogonal projection.

Proof. Assume $\left\{e_{n}\right\}_{n=0}^{\infty}$ is effective. By (5) and by the polar identity we get

$$
\langle x, y\rangle=\sum_{n=0}^{\infty}\left\langle x, g_{n}\right\rangle\left\langle g_{n}, y\right\rangle
$$

for any $x, y \in \mathcal{H}$. In particular

$$
m_{i j}=\left\langle e_{i}, e_{j}\right\rangle=\sum_{n=0}^{\infty}\left\langle e_{i}, g_{n}\right\rangle\left\langle g_{n}, e_{j}\right\rangle .
$$

We want to state the formula (10) in terms of matrices on $\ell^{2}\left(\mathbb{N}_{0}\right)$. Let $\delta_{i}$ denote the sequence in $\ell^{2}\left(\mathbb{N}_{0}\right)$ whose $i$ th entry is 1 and all other entries are 0 . We have the following.

LEMMA 1.

$$
\left\langle g_{n}, e_{j}\right\rangle=\left\langle\left(U M^{*}+M^{*}+I\right) \delta_{j}, \delta_{n}\right\rangle_{\ell^{2}\left(\mathbb{N}_{0}\right)}
$$

Proof of Lemma 1. Set $c_{n n}=1$. Then by (6) we have

$$
\left\langle g_{n}, e_{j}\right\rangle= \begin{cases}\sum_{i=0}^{n} c_{n i} m_{i j} & \text { for } j>n, \\ j-1 & \text { for } j \leq n . \\ \sum_{i=0} c_{n i} m_{i j}+\sum_{i=j}^{n} c_{n i} m_{i j} & \end{cases}
$$


Since $(I+U)(I+M)=I$ we get

$$
\sum_{i=j}^{n} c_{n i} m_{i j}=\delta_{j n}, \quad \text { for } j \leq n .
$$

Therefore

$$
\begin{aligned}
\left\langle g_{n}, e_{j}\right\rangle & =\left\langle\sum_{i=0}^{j-1} \bar{m}_{j i} \delta_{i}, \sum_{i=0}^{n} \bar{c}_{n i} \delta_{i}\right\rangle_{\ell^{2}}+\left\langle\delta_{j}, \delta_{n}\right\rangle_{\ell^{2}} \\
& =\left\langle M^{*} \delta_{j},\left(U^{*}+I\right) \delta_{n}\right\rangle_{\ell^{2}}+\left\langle\delta_{j}, \delta_{n}\right\rangle_{\ell^{2}}=\left\langle\left(U M^{*}+M^{*}+I\right) \delta_{j}, \delta_{n}\right\rangle_{\ell^{2}}
\end{aligned}
$$

Let $A=U M^{*}+M^{*}+I$. Applying Lemma 1 to (10) and using the Parseval identity gives

$$
m_{i j}=\sum_{n=0}^{\infty}\left\langle A \delta_{j}, \delta_{n}\right\rangle\left\langle\delta_{n}, A \delta_{i}\right\rangle_{\ell^{2}}=\left\langle A \delta_{j}, A \delta_{i}\right\rangle_{\ell^{2}} .
$$

Let $A_{n}=U_{n} M_{n}^{*}+M_{n}^{*}+I$. Unlike $A$, the matrices $A_{n}$ correspond to bounded operators on $\ell^{2}\left(\mathbb{N}_{0}\right)$. Since $M_{n}^{*} \delta_{k}=M^{*} \delta_{k}$ for $n \geq k$ and $U_{n} \stackrel{n}{\rightarrow} U$ strongly we have

$$
\lim _{n \rightarrow \infty}\left\langle A_{n}^{*} A_{n} \delta_{j}, \delta_{i}\right\rangle_{\ell^{2}}=\lim _{n \rightarrow \infty}\left\langle A_{n} \delta_{j}, A_{n} \delta_{i}\right\rangle_{\ell^{2}}=\left\langle A \delta_{j}, A \delta_{i}\right\rangle_{\ell^{2}} .
$$

On the other hand, the relation $M_{n} U_{n}=-U_{n}-M_{n}$ implies

$$
\begin{aligned}
A_{n}^{*} A_{n}= & M_{n} U_{n}^{*} U_{n} M_{n}^{*}-M_{n}\left(U_{n}^{*}+M_{n}^{*}\right)+M_{n} U_{n}^{*} \\
& -\left(U_{n}+M_{n}\right) M_{n}^{*}+M_{n} M_{n}^{*}+M_{n}+U_{n} M_{n}^{*}+M_{n}^{*}+I \\
= & M_{n}+M_{n}^{*}+I+M_{n} U_{n}^{*} U_{n} M_{n}^{*}-M_{n} M_{n}^{*} .
\end{aligned}
$$

Hence

(13) $\lim _{n \rightarrow \infty}\left\langle A_{n}^{*} A_{n} \delta_{j}, \delta_{i}\right\rangle_{\ell^{2}}=m_{i j}+\left(U M^{*} \delta_{j}, U M^{*} \delta_{i}\right)_{\ell^{2}}-\left(M^{*} \delta_{j}, M^{*} \delta_{i}\right)_{\ell^{2}}$.

Combining (11)-(13) yields

$$
\left(U M^{*} \delta_{j}, U M^{*} \delta_{i}\right)_{\ell^{2}}=\left(M^{*} \delta_{j}, M^{*} \delta_{i}\right)_{\ell^{2}} .
$$

Let $\mathcal{F}\left(\mathbb{N}_{0}\right)=\operatorname{span}\left\{\delta_{0}, \delta_{1}, \ldots\right\}$. Formula (14) states that the operator $U$ is isometric on

$$
\mathcal{H}_{0}=\overline{M^{*}\left(\mathcal{F}\left(\mathbb{N}_{0}\right)\right)} .
$$

It suffices to show that $U$ vanishes on $\mathcal{H}_{0}^{\perp}$. To this end observe that the matrices $U^{*}$ and $M^{*}$ leave the subspace $\mathcal{F}\left(\mathbb{N}_{0}\right)$ invariant. The formula $M^{*}\left(U^{*}+I\right)$ $=-U^{*}$ implies that

$$
U^{*}\left(\mathcal{F}\left(\mathbb{N}_{0}\right)\right) \subset \mathcal{H}_{0} .
$$

Taking orthogonal complements of both sides results in

$$
\mathcal{H}_{0}^{\perp} \subset \operatorname{ker} U
$$

which completes the proof that $U$ is a partial isometry. 
Conversely, let $U$ be a partial isometry. Hence $U$ is isometric on $\mathcal{H}_{0}=$ $\overline{U^{*}\left(\mathcal{F}\left(\mathbb{N}_{0}\right)\right)}$. The formula $U^{*}\left(M^{*}+I\right)=-M^{*}$ implies that $U$ is isometric on $M^{*}\left(\mathcal{F}\left(\mathbb{N}_{0}\right)\right)$, which is equivalent to (14). Now tracking backwards the proof of the first part implies the formula (10). In particular for $i=j$ we obtain

$$
\left\|e_{i}\right\|^{2}=\sum_{n=0}^{\infty}\left|\left\langle e_{i}, g_{n}\right\rangle\right|^{2}
$$

for any $i \geq 0$. This implies effectivity (see comments at the end of the Introduction).

REMARK 1. Theorem 1 can be used to construct examples of effective sequences of vectors. It suffices to come up with a strictly lower triangular partial isometry $U$. Then one has to compute the algebraic inverse $I+M$ of $I+U$. As a result a positive definite matrix $M+M^{*}+I$ is constructed. The corresponding vectors form a sequence effective in the closed linear span of these vectors.

Remark 2. Proposition 1 and Theorem 1 can be interpreted as follows. There are as many effective sequences among sequences of unit vectors as there are partial isometries among strictly lower triangular contractions on $\ell^{2}\left(\mathbb{N}_{0}\right)$. This can be compared with a result of Kwapien and Mycielski who showed that if we choose the sequence of unit vectors at random then almost surely we end up with an effective sequence. More precisely, fix a probability Borel measure $\mu$ on the unit sphere of $\mathcal{H}$, such that the support of $\mu$ is linearly dense. Then drawing consecutive vectors independently with respect to that measure almost surely yields an effective sequence of vectors.

3. Strongly effective sequences. For a partial isometry $U$ the carrier space, i.e. $\operatorname{Im} U^{*}$, may vary from the trivial space to the whole space $\mathcal{H}$. For instance when the system $\left\{e_{n}\right\}_{n=0}^{\infty}$ is orthonormal we get $M=U=0$. The other extreme case is when $\operatorname{Im} U^{*}=\mathcal{H}$, which is equivalent to $U^{*} U=I$. While proving Theorem 1 we showed that for an effective sequence $\left\{e_{n}\right\}_{n=0}^{\infty}$ the carrier space for $U$ coincides with $\overline{M^{*}\left(\mathcal{F}\left(\mathbb{N}_{0}\right)\right)}$. Hence $U^{*} U=I$ if and only if $\overline{M^{*}\left(\mathcal{F}\left(\mathbb{N}_{0}\right)\right)}=\ell^{2}\left(\mathbb{N}_{0}\right)$. This occurs exactly when the rows of the matrix $M$ form a linearly dense subset of $\ell^{2}\left(\mathbb{N}_{0}\right)$. For example this is the case when $m_{n+1, n} \neq 0$ in the matrix

$$
\left(\begin{array}{ccccc}
0 & & & & \\
m_{10} & 0 & & & \\
* & m_{21} & 0 & & \\
* & * & m_{32} & 0 & \\
\vdots & \vdots & & \ddots & \ddots
\end{array}\right) .
$$


It turns out that sequences $\left\{e_{n}\right\}_{n=0}^{\infty}$ with $U^{*} U=I$ have many interesting properties. Let

$$
v_{i n}=\sum_{j=i}^{n} \bar{c}_{n j} e_{j}, \quad n>i .
$$

The next lemma is related to a formula obtained in the proof of Theorem 2 of $[2]$.

LEMMA 2. For $0 \leq i, j<n$ the following equalities hold:

$$
\left\langle v_{i n}, v_{j n}\right\rangle=\left\langle\left(I-U_{n}^{*} U_{n}\right) \delta_{j}, \delta_{i}\right\rangle_{\ell^{2}} .
$$

Proof. Without loss of generality we may assume that $j \leq i$. Then using the fact that $I+M$ and $I+U$ are inverse to each other we get

$$
\begin{aligned}
\left\langle v_{i n}, v_{j n}\right\rangle & =\left\langle\sum_{k=i}^{n} \bar{c}_{k i} e_{k}, \sum_{l=j}^{n} \bar{c}_{l j} e_{l}\right\rangle=\sum_{k=i}^{n} \bar{c}_{k i} \sum_{l=j}^{n} m_{k l} c_{l j}=\sum_{k=i}^{n-1} \bar{c}_{k i} \sum_{l=j}^{n} m_{k l} c_{l j} \\
& =\sum_{k=i}^{n-1} \bar{c}_{k i} \sum_{l=j}^{k} m_{k l} c_{l j}+\sum_{k=i}^{n-1} \bar{c}_{k i} \sum_{l=k+1}^{n} m_{k l} c_{l j} \\
& =\sum_{k=i}^{n-1} \bar{c}_{k i} \delta_{j k}+\sum_{l=i+1}^{n} c_{l j} \sum_{k=i}^{l-1} \bar{m}_{l k} \bar{c}_{k i}=\delta_{i j}-\sum_{l=i+1}^{n} c_{l j} \bar{c}_{l i} \\
& =\left\langle\left(I-U_{n}^{*} U_{n}\right) \delta_{j}, \delta_{i}\right\rangle_{\ell^{2}} .
\end{aligned}
$$

The next corollary should be compared with Remark 2 of [2].

Corollary 1. Assume $U^{*} U=I$. Then for any $j \geq 0$,

$$
e_{j}=-\sum_{i=j+1}^{\infty} \bar{c}_{i j} e_{i}
$$

Proof. By Lemma 2 we get $v_{j n} \rightarrow 0$ as $n \rightarrow \infty$. Hence

$$
\sum_{i=j}^{\infty} \bar{c}_{i j} e_{i}=0 .
$$

Since $c_{j j}=1$ we get the conclusion.

DEFINITION 1. The sequence $\left\{e_{n}\right\}_{n=0}^{\infty}$ will be called strongly effective if $\left\{e_{n}\right\}_{n=k}^{\infty}$ is effective for each $k \geq 0$.

In particular dropping finitely many vectors from $\left\{e_{n}\right\}_{n=0}^{\infty}$ does not spoil linear density. Hence this is a highly nonorthogonal case.

THEOREM 2. Assume the sequence $\left\{e_{n}\right\}_{n=0}^{\infty}$ is linearly dense in a Hilbert space $\mathcal{H}$. Then $\left\{e_{n}\right\}_{n=0}^{\infty}$ is strongly effective if and only if $U^{*} U=I$. 
Proof. By Corollary 1 for any $k$ the sequence $\left\{e_{n}\right\}_{n=k}^{\infty}$ is linearly dense. Let $M^{(k)}$ and $U^{(k)}$ denote truncated matrices obtained by removing the first $k$ rows and the first $k$ columns from $M$ and $U$, respectively. These matrices correspond to the sequence $\left\{e_{n}\right\}_{n=k}^{\infty}$. Also $U^{*} U=I$ implies $\left(U^{(k)}\right)^{*} U^{(k)}=I$ for any $k$. Hence $U^{(k)}$ is a partial isometry. Now we can use Theorem 1 to get the conclusion.

Conversely, suppose that $\left\{e_{n}\right\}_{n=0}^{\infty}$ is strongly effective. Let $Q_{k}$ denote the orthogonal projection from $\ell^{2}(\mathbb{N})$ onto the orthogonal complement of $\left\{\delta_{0}, \delta_{1}, \ldots, \delta_{k-1}\right\}$. Let $U_{(k)}=U Q_{k}$. Then $U_{(k)}=0_{k} \oplus U^{(k)}$, where $0_{k}$ denotes the $k \times k$ zero matrix. Hence $U_{(k)}$ are partial isometries just as $U$ is. But this is possible only if $U^{*} U$ and $Q_{k}$ commute. On the other hand, if $U^{*} U$ commutes with $Q_{k}$ for any $k$ then $U^{*} U$ must be diagonal. Assume that $U^{*} U \neq I$. Then $U \delta_{j}=0$ for some $j$. This implies that $M \delta_{j}=0$ and consequently $e_{j}$ is orthogonal to all the vectors $e_{i}, i>j$. Hence $\left\{e_{n}\right\}_{n=0}^{\infty}$ cannot be strongly effective.

Lemma 3. Assume $U^{*} U=I$. Then

$$
\begin{aligned}
U^{*} M & =-M-I, \\
U^{*}\left(M+M^{*}+I\right) & =-\left(M+M^{*}+I\right) .
\end{aligned}
$$

Proof. By taking the inner product with $e_{k}$ in (16) we obtain

$$
m_{j k}=-\sum_{i=j+1}^{\infty} \bar{c}_{i j} m_{i k}
$$

for any $k \geq 0$. Let $k \leq j$. Then

$$
m_{j k}=-\sum_{i=j+1}^{\infty} \bar{c}_{i j} m_{i k}=\left(U^{*} M\right)_{j k} .
$$

For $k>j$ we get

$$
\begin{aligned}
0 & =\sum_{i=j}^{\infty} \bar{c}_{i j} m_{i k}=\sum_{i=j}^{k} \bar{c}_{i j} \bar{m}_{k i}+\sum_{i=k+1}^{\infty} \bar{c}_{i j} m_{i k} \\
& =\overline{((M+I) U)_{j k}}+\left(U^{*} M\right)_{j k}=\left(U^{*} M\right)_{j k} .
\end{aligned}
$$

Combining (18) and (19) gives the first equality. The second equality now follows by applying $U^{*} M^{*}=-U^{*}-M^{*}$.

Theorem 3. Assume the matrix $U$ associated with the sequence $\left\{e_{n}\right\}_{n=0}^{\infty}$ satisfies $U^{*} U=I$. Then

$$
\sum_{i=0}^{\infty}\left|\left\langle e_{i}, e_{j}\right\rangle\right|^{2}=\infty
$$


for any $j$. In particular the conclusion holds if the sequence $\left\{e_{n}\right\}_{n=0}^{\infty}$ is effective and the rows of $M$ are linearly dense in $\ell^{2}\left(\mathbb{N}_{0}\right)$.

Proof. Let $G=M+M^{*}+I$. Observe that $G$ is nothing other than the Gram matrix of the vectors $\left\{e_{n}\right\}_{n=0}^{\infty}$. Assume that

$$
\sum_{i=0}^{\infty}\left|\left\langle e_{i}, e_{j}\right\rangle\right|^{2}<\infty
$$

for some $j$. Then $v=G \delta_{j} \in \ell^{2}\left(\mathbb{N}_{0}\right)$. By (17) we get $U^{*} v=-v$ and

$$
\left(U^{*}\right)^{n} v=(-1)^{n} v \text {. }
$$

But $\left\|U^{*}\right\| \leq 1$ and $U^{*}$ is strictly upper triangular. Hence $\left(U^{*}\right)^{n}$ tends to zero strongly, which implies $v=0$. This gives a contradiction because $v(j)=1$.

4. Stationary case. Assume

$$
\left\langle e_{i+1}, e_{j+1}\right\rangle=\left\langle e_{i}, e_{j}\right\rangle \text {. }
$$

Then the matrix $M$ is constant on diagonals:

$$
M=\left(\begin{array}{ccccc}
0 & & & & \\
a_{1} & 0 & & & \\
a_{2} & a_{1} & 0 & & \\
a_{3} & a_{2} & a_{1} & 0 & \\
\vdots & \vdots & \vdots & \vdots & \ddots
\end{array}\right)
$$

By the Herglotz theorem there is a measure $\mu$ on the unit circle such that

$$
\left\langle e_{i+n}, e_{i}\right\rangle=a_{n}=\int_{\mathbb{T}} z^{n} d \mu(z) .
$$

Kwapień and Mycielski showed that the sequence $\left\{e_{n}\right\}_{n=0}^{\infty}$ is effective if and only if either $\mu$ is the Lebesgue measure (orthogonal case) or it is singular with respect to the Lebesgue measure. We now reprove this result by applying our Theorem 1.

Also $U$ is constant on diagonals, i.e. it is a Toeplitz operator,

$$
U=\left(\begin{array}{cccccc}
0 & & & & \\
u_{1} & 0 & & & \\
u_{2} & u_{1} & 0 & & \\
u_{3} & u_{2} & u_{1} & 0 & \\
\vdots & \vdots & \vdots & \vdots & \ddots
\end{array}\right)
$$

It is then unitarily equivalent to the multiplication operator on $H^{2}(\mathbb{T})$ with the function

$$
u(z)=\sum_{n=1}^{\infty} u_{n} z^{n} .
$$




\section{Moreover}

$$
\|U\|=\|u(z)\|_{H^{\infty}(\mathbb{T})} \leq 1 .
$$

Now multiplication with $u(z)$ is a partial isometry if and only if the boundary values of $|u(z)|$ are 0 or 1 . By the F. Riesz and M. Riesz theorem, $u\left(e^{i \theta}\right) \equiv 0$ or $\left|u\left(e^{i \theta}\right)\right| \equiv 1$. The first case corresponds to the orthogonal case, because $M=0$. The second case is equivalent to the singularity of the spectral measure $\mu$. Indeed, for $a(z)=\sum_{n=1}^{\infty} a_{n} z^{n}$ we have $(a(z)+1)(u(z)+1)=1$. Therefore

$$
1+a(z)+\overline{a(z)}=\frac{1-|u(z)|^{2}}{|u(z)+1|^{2}} .
$$

By the Fatou theorem

$$
\lim _{r \rightarrow 1^{-}}\left[1+a\left(r e^{i \theta}\right)+\overline{a\left(r e^{i \theta}\right)}\right]=\frac{d \mu}{d \theta} .
$$

Therefore $\mu$ is singular if and only if $\left|u\left(e^{i \theta}\right)\right| \rightarrow 1$ almost everywhere.

Acknowledgements. The first named author wishes to acknowledge that this work was done during his fellowship stay at the University of Wrocław in 2003-2004. He would like to thank warmly Prof. E. Damek and Prof. R. Szwarc for their hospitality.

\section{References}

[1] S. Kaczmarz, Approximate solution of systems of linear equations, Bull. Acad. Polon. Sci. Lett. A 35 (1937), 355-357 (in German); English transl.: Internat. J. Control 57 (1993), 1269-1271.

[2] S. Kwapień and J. Mycielski, On the Kaczmarz algorithm of approximation in infinitedimensional spaces, Studia Math. 148 (2001), 75-86.

Institute of Pure Mathematics

Faculty of Mathematics and Computer Science

University of Tartu

Ülikooli 18

50090 Tartu, Estonia

E-mail: Rainis.Haller@ut.ee
Institute of Mathematics

University of Wrocław

Pl. Grunwaldzki 2/4

50-384 Wrocław, Poland

E-mail: szwarc@math.uni.wroc.pl

Received July 21, 2004

Revised version February 24, 2005 